\title{
REALIZATION OF THE LANDAU DEFINITIONS OF EFFECTIVE HAMILTONIAN AND NONEQUILIBRIUM FREE ENERGY IN MICROSCOPIC THEORY
}

\author{
A.I. Sokolovsky \\ Oles Honchar Dnipro National University, Dnipro, Ukraine \\ *e-mail: alexander.i.sokolovsky@gmail.com
}

Equilibrium fluctuations of some set of parameters in the states described by the canonical Gibbs distribution are investigated. In the theory of phase transitions of the second kind, these parameters are components of the order parameter. The microscopic realization of the Landau definition of the effective Hamiltonian of the system for studying the equilibrium fluctuations of the specified system of parameters is discussed in the terms of the probability density of their values. A general formula for this function is obtained and it is expressed through the equilibrium correlation functions of these parameters. An expression for the effective Hamiltonian in terms of deviations of the parameters from their equilibrium values is obtained. The deviations are considered small for conducting the calculations. The possibility of calculating the exact free energy of the system using the found effective Hamiltonian is discussed.

In the microscopic theory, the implementation of the Landau definition of nonequilibrium thermodynamic potentials introduced in his phenomenological theory of phase transitions of the second kind is investigated. Nonequilibrium states of a fluctuating system described with some sets of parameters are considered. A general formula for nonequilibrium free energy expressed through the correlation functions of these parameters is obtained as for the effective Hamiltonian above. Like the previous case, the free energy expression via parameter deviations from the equilibrium values is obtained and small deviations are considered for calculations. The idea of the identity of the effective Hamiltonian of the system and its nonequilibrium free energy is discussed in connection with the Boltzmann distribution. The Gaussian approximation of both developed formalisms is considered.

A generalization of the constructed theory for the case of spatially inhomogeneous states and the study of long-wave fluctuations are developed.

Keywords: equilibrium fluctuations, effective Hamiltonian, nonequilibrium free energy, the Boltzmann principle, phase transitions of the second kind, correlation functions, fluctuations close to equilibrium, long-wave fluctuations.

Received 25.10.2020; Received in revised form 28.11.2020; Accepted 02.12.2020

\section{Introduction}

The investigation of equilibrium fluctuations is a classic problem of statistical mechanics [1]. They are due to the fact that the observed values of physical quantities differ from their average values, because even the equilibrium system is dynamic one. To some extent, fluctuations depend on the conditions in which the system is. For certainty, we consider a system whose equilibrium state is described by the canonical Gibbs distribution

$$
w=e^{\frac{F-\hat{H}}{T}}, \quad \operatorname{Sp} w=1 .
$$

The system has a fixed temperature $T$, volume $V$, external fields, and the number of particles of all components. Hereinafter, the microscopic value of a physical quantity $\hat{a}$ as a function of the coordinates and momentums of the particles is denoted by a hat. The integral over the phase space together with the required factors is denoted by the symbol Sp. This shortens the notation of formulas and brings together the notation of classical and quantum theory, in which the physical quantity operator is denoted by $\hat{a}$.

The free energy of the system is determined from the normalization condition (1). The average values of physical quantities are calculated by the formula

$$
\bar{a}=\mathrm{Sp} w \hat{a} .
$$

Let us set the problem of studying the fluctuations of some system of physical quantities $\eta_{a}$ 
(the index $a$ and then the indices $a, b, c$ number these quantities). In the theory of phase transitions of the second kind, the components of the order parameter are chosen as these parameters. These fluctuations are completely described by the function of their distribution [1]

$$
\begin{array}{cc}
w(\eta)=\operatorname{Sp} w \delta(\eta-\hat{\eta}), & \delta(\eta-\hat{\eta}) \equiv \prod_{a} \delta\left(\eta_{a}-\hat{\eta}_{a}\right) ; \\
\int d \eta w(\eta)=1, & \int d \eta=\prod_{a} \int_{-\infty}^{\infty} d \eta_{a},
\end{array}
$$

since the formula

$$
\overline{\mathrm{f}(\hat{\eta})}=\operatorname{Sp} w \mathrm{f}(\hat{\eta})=\int d \eta w(\eta) \mathrm{f}(\eta) .
$$

is valid for an arbitrary function $\mathrm{f}(\eta)$.

The fluctuation state of the system can be considered as a nonequilibrium state, which is described by the parameters of the reduced description $\eta_{a}$. The Boltzmann formula

$$
w(\eta)=A e^{-\frac{F(\eta)}{T}}
$$

is traditionally associated with the function $w(\eta)[1,2]$. Here $F(\eta)$ is the free energy of the system in the specified nonequilibrium state ( $A$ is normalization factor). The function $F(\eta)$ is also called the effective Hamiltonian in the space of parameters $\eta_{a}$ [2]. The definition of nonequilibrium thermodynamic potential was introduced by Landau in his theory of phase transitions of the second kind as some equilibrium thermodynamic potential for which the order parameters are eigenvariables $[1,3,4]$. It was considered clear that this could be done by a Legendre transformation of thermodynamic potential in the presence of an external field $[4,5]$. This explains why Landau did not use the term "nonequilibrium thermodynamic potential". A little later after Landau, this idea of introducing nonequilibrium thermodynamic potential was proposed by Leontovich [6] (see also [7]) on the basis of the idea that the nonequilibrium state of a system can be considered as an equilibrium one in the presence of some external field.

According to Pitaevskii (see [1]), the definition of the effective Hamiltonian by the formula $H(\eta)$

$$
w(\eta)=e^{\frac{F-H(\eta)}{T}}
$$

belongs to Landau. Here $F$ is the exact free energy of the system, and the function $w(\eta)$ is defined in (3). Formula (5) is a complete analogue of the canonical Gibbs distribution (1), which describes the system in phase space. It can be used according to Landau [1] to calculate the free energy of the system.

Formulas (1), (3), and (5) give the following expression for the effective Landau Hamiltonian $H(\eta)$

$$
H(\eta)=-T \ln \operatorname{Sp} e^{-\frac{\hat{H}}{T}} \delta(\eta-\hat{\eta}),
$$

which is directly proposed by Landau (see [1]).

64 
Note that the present work is a continuation of our papers $[8,9]$. The first of them [8] was devoted to the development of an expression for nonequilibrium free energy, based on the ideas of Leontovich's work [6,7]. Our current paper describes the course of Landau's ideas in connection with the introduction of nonequilibrium thermodynamic potentials. The second work [9] is devoted to the construction of an expression for an effective Hamiltonian of the system $H(\eta)$. In the present work, the derivation of the basic formula for the probability density is simplified and an in-depth analysis of the Hamiltonian $H(\eta)$ construction problem is performed.

Consideration of these problems was largely stimulated by the development of the theory of phase transitions of the second kind, the main provisions of which were developed by Landau [1, 2]. It should be noted that this theory is mainly phenomenological (see, for example, [2, 10, 11]). However, some studies are devoted to the development of microscopic theory and in particular the calculation of nonequilibrium free energy. See a thorough review of the microscopic theory in the vicinity of the critical point of a liquid-vapor system in [12].

The structure of the work is as follows. Section 2 is devoted to the implementation of the definition of the Landau effective Hamiltonian of a system in the microscopic theory. Section 3 gives a microscopic theory of the construction of nonequilibrium free energy of the system based on its Landau definition. Section 4 proposes generalization of the developed theory for non-homogeneous states and long-wave fluctuations.

\section{The effective Landau Hamiltonian}

Let us start with the calculation of the equilibrium distribution function $w(\eta)$ of fluctuations of some parameters $\eta_{a}$ in the vicinity of the equilibrium state of the system. Given the expansion of the $\delta$-functions in the Fourier integral, from formulas (1) and (3) we have

$$
\begin{aligned}
w(\eta) & =\operatorname{Sp} w \delta(\eta-\hat{\eta})=\frac{1}{(2 \pi)^{s}} \int d u \operatorname{Sp} w e^{i \sum_{a} u_{a}\left(\eta_{a}-\hat{\eta}_{a}\right)}=, \\
& =\frac{1}{(2 \pi)^{s}} e^{\frac{F}{T}} \int d u e^{i \sum_{a} u_{a} \eta_{a}} \operatorname{Sp} e^{-\frac{\hat{H}+\sum_{a} i u_{a} T \hat{\eta}_{a}}{T}}
\end{aligned}
$$

where is denoted

$$
d u=\prod_{a} d u_{a}, \int d u=\prod_{a} \int_{-\infty}^{\infty} d u_{a}
$$

( $s$ is the number of the $\eta_{a}$ ).

For further transformations, it is convenient to use the canonical Gibbs distribution in the presence of an external field $h_{a}$ introduced through the help of microscopic variables $\hat{\eta}_{a}$ that correspond to the parameters $\eta_{a}$

$$
w(h)=e^{\frac{F(h)-\left[\hat{H}+\sum_{a} h_{a} \hat{\eta}_{a}\right]}{T}}, \quad \operatorname{Sp} w(h)=1 .
$$

In thermodynamics, different sets of independent variables can be selected. It is convenient for us to consider the free energy $F(h)$ and the canonical Gibbs distribution 
$w(h)$ in the presence of a field as a function of the field $h_{a}$. Hence for the trace in formula (8) such an expression through the free energy $F(h)$ in the field presence is found

$$
\mathrm{Sp} e^{-\frac{\hat{H}+\sum_{a} i u_{a} T \hat{\eta}_{a}}{T}}=e^{-\frac{F(i h T)}{T}} .
$$

Taking into account (11), expression (8) for the probability density $w(\eta)$ of the parameters $\eta_{a}$ takes the form

$$
w(\eta)=\frac{1}{(2 \pi)^{s}} \int d u e^{i \sum_{a} u_{a} \eta_{a}} e^{\frac{F-F(i u T)}{T}}
$$

In our previous work [8] it was observed that the free energy $F(h)$ in the presence of an external field can be expressed through a generating function $\mathbb{F}(h)$ for the mean values of the products of quantities $-\hat{\eta}_{a} / T$. Indeed, from (1) and (10) the formula

$$
e^{-\frac{F(h)}{T}}=\operatorname{Sp} e^{-\frac{\hat{H}}{T}} e^{-\sum_{a} h_{a} \frac{\hat{\eta}_{a}}{T}}=e^{-\frac{F}{T}} \operatorname{Sp} w e^{-\sum_{a} h_{a} \frac{\hat{\eta}_{a}}{T}}
$$

follows, that is

$$
e^{\frac{F-F(h)}{T}}=\mathbb{F}(h)
$$

where the generating function $\mathbb{F}(h)$ is introduced for the average values of the products of quantities $-\eta_{a} / T$ in the absence of an external field

$$
\mathbb{F}(h) \equiv e^{\overline{-\sum_{a} h_{a} \frac{\hat{\eta}_{a}}{T}}}=1+\sum_{n=1}^{\infty} \frac{(-1)^{n}}{n ! T^{n}} \sum_{a_{1} \ldots a_{n}} h_{a_{1}} \ldots h_{a_{n}} \overline{\hat{\eta}_{a_{1}} \ldots \hat{\eta}_{a_{n}}} .
$$

Instead of averages $\overline{\hat{\eta}_{a_{1}} \ldots \hat{\eta}_{a_{n}}}$, it is convenient to introduce correlation functions $\left\langle\hat{\eta}_{a_{1}} \ldots \hat{\eta}_{a_{n}}\right\rangle$, which are determined sequentially by formulas

$$
\begin{gathered}
\overline{\hat{\eta}_{a_{1}} \hat{\eta}_{a_{2}}}=\overline{\hat{\eta}_{a_{1}}} \overline{\hat{\eta}_{a_{2}}}+\left\langle\hat{\eta}_{a_{1}} \hat{\eta}_{a_{2}}\right\rangle, \\
\overline{\hat{\eta}_{a_{1}} \hat{\eta}_{a_{2}} \hat{\eta}_{a_{3}}}=\overline{\hat{\eta}_{a_{1}}} \overline{\hat{\eta}_{a_{2}}} \overline{\hat{\eta}_{a_{3}}}+\left\langle\hat{\eta}_{a_{1}} \hat{\eta}_{a_{2}}\right\rangle \overline{\hat{\eta}_{a_{3}}}+\left\langle\hat{\eta}_{a_{2}} \hat{\eta}_{a_{3}} \overline{\hat{\eta}_{a_{1}}}+\left\langle\hat{\eta}_{a_{1}} \hat{\eta}_{a_{3}}\right\rangle \overline{\hat{\eta}_{a_{2}}}+\right. \\
+\left\langle\hat{\eta}_{a_{1}} \hat{\eta}_{a_{2}} \hat{\eta}_{a_{3}}\right\rangle
\end{gathered}
$$

and so on. The generating function for correlation functions of quantities $-\hat{\eta}_{a} / T$

$$
\mathbb{G}(h) \equiv \sum_{n=2}^{\infty} \frac{(-1)^{n}}{n ! T^{n}} \sum_{a_{1} \ldots a_{n}} h_{a_{1}} \ldots h_{a_{n}}\left\langle\hat{\eta}_{a_{1}} \ldots \hat{\eta}_{a_{n}}\right\rangle,
$$

is related with the generating function of the averages by the formula 66 


$$
\mathbb{F}(h)=\exp \left[-\sum_{a} h_{a} \frac{\eta_{a 0}}{T}+\mathbb{G}(h)\right] \quad\left(\eta_{a 0} \equiv \overline{\hat{\eta}_{a}}\right)
$$

(see in $[13,14]$ pages 116 and 12, respectively). Formulas (14) and (18) give the following expression for the free energy of the system in the presence of the external field $h_{a}$

$$
F(h)=F+\sum_{a} h_{a} \eta_{a 0}-T \mathbb{G}(h)
$$

(obtained in our work [8]). Given this relationship, formula (12) for the fluctuation distribution function acquires the final form

$$
w(\eta)=\frac{1}{(2 \pi)^{s}} \int d u e^{\mathbb{G}(i u T)} e^{i \sum_{a} u_{a} \delta \eta_{a}}, \quad \delta \eta_{a} \equiv \eta_{a}-\eta_{a 0} .
$$

Based on this formula taking into account definition (5), one can find the effective Hamiltonian $H(\eta)$ and distribution $w(\eta)$ for states in the vicinity of the equilibrium state

$$
\begin{array}{rlrl}
H(\eta) & =F-T \ln w(\eta), & w(\eta) & =A_{0}+\sum_{n=1}^{+\infty} \sum_{a_{1} \ldots a_{n}} A_{a_{1} \ldots a_{n}} \delta \eta_{a_{1}} \ldots \delta \eta_{a_{n}}, \\
A_{0} & \equiv \frac{1}{(2 \pi)^{s}} \int d u e^{\mathbb{G}(i u T)}, & A_{a_{1} \ldots a_{n}} \equiv \frac{i^{n}}{(2 \pi)^{s} n !} \int d u e^{\mathbb{G}(i u T)} u_{a_{1}} \ldots u_{a_{n}}
\end{array}
$$

(these expressions were not obtained in our work [9]). Taking into account the Taylor expansion

$$
\begin{gathered}
\ln \left[b_{0}+b_{1} x+b_{2} x^{2}+b_{3} x^{3}+b_{4} x^{4}+O\left(x^{5}\right)\right]= \\
=\ln b_{0}+\frac{b_{1}}{b_{0}} x+\left(\frac{b_{2}}{b_{0}}-\frac{b_{1}^{2}}{2 b_{0}^{2}}\right) x^{2}+\left(\frac{b_{3}}{b_{0}}-\frac{b_{1} b_{2}}{b_{0}^{2}}+\frac{b_{1}^{3}}{3 b_{0}^{3}}\right) x^{3}+ \\
+\left(\frac{b_{4}}{b_{0}}-\frac{b_{2}^{2}}{2 b_{0}^{2}}-\frac{b_{1} b_{3}}{b_{0}^{2}}+\frac{b_{1}^{2} b_{2}}{b_{0}^{3}}-\frac{b_{1}^{4}}{4 b_{0}^{4}}\right) x^{4}+O\left(x^{5}\right),
\end{gathered}
$$

one can get an effective Hamiltonian with the desired accuracy. Particularly, in the Gaussian approximation we have

$$
\begin{gathered}
H(\eta)=F-T\left[\ln A_{0}+\frac{1}{A_{0}} \sum_{a} A_{a} \delta \eta_{a}-\frac{1}{2} \sum_{a, b} D_{a b} \delta \eta_{a} \delta \eta_{b}+O\left(\delta \eta^{3}\right)\right], \\
D_{a b} \equiv \frac{2}{A_{0}}\left(\frac{1}{2 A_{0}} A_{a} A_{b}-A_{a b}\right)
\end{gathered}
$$


(here notations are somewhat simplified: $a_{1} \rightarrow a, a_{2} \rightarrow b$ ). Formula (21)

simultaneously gives the following expression for the probability density $w(\eta)$ of parameter values $\eta_{a}$

$$
w(\eta)=A_{0} \exp \left[\frac{1}{A_{0}} \sum_{a} A_{a} \delta \eta_{a}-\frac{1}{2} \sum_{a, b} D_{a b} \delta \eta_{a} \delta \eta_{b}+O\left(\delta \eta^{3}\right)\right] .
$$

Expression (24) shows that the most probable values $\eta_{a}$ of the parameters $\eta_{a}$ differ due to fluctuations from their average values $\eta_{a 0}$ by some quantity $\Delta_{a}$

$$
\begin{aligned}
& \delta \eta_{a}=\delta \gamma_{a}+\Delta_{a}, \quad \delta \eta_{a}=\eta_{a}-\eta_{a 0}, \quad \delta \gamma_{a}=\eta_{a}-\eta_{a}^{o}, \\
& \sum_{a} A_{a} \delta \eta_{a}+\sum_{a, b} D_{a b} \delta \eta_{a} \delta \eta_{b}=\sum_{a, b} D_{a b} \delta \eta_{a} \delta \eta_{q_{b}}+ \\
& +\sum_{a} A_{a} \Delta_{a}+\sum_{a, b} D_{a b} \Delta_{a} \Delta_{b}, \quad \Delta_{a} \equiv \frac{1}{A_{0}} \sum_{b} D_{a b}^{-1} A_{b}
\end{aligned}
$$

(the transition from $\delta \eta_{a}$ to $\delta f_{a}$ zeroes the linear in $\delta f f_{a}$ contributions). As a result, the probability density in the Gaussian approximation is given by the formula

$$
w_{2}(\eta)=\left[\frac{\operatorname{det} D}{(2 \pi)^{s}}\right]^{1 / 2} \exp \left[-\frac{1}{2} \sum_{a, b} D_{a b} \delta f f_{a} \delta f_{b}\right] .
$$

It should be noted that function (24) is normalized to 1 only taking into account the whole series by powers $\delta \eta_{a}$ (see (20)). In expression (26), compared with (24), the normalization factor in the probability density is changed, because in (26) the series in $\delta \eta_{a}$ degrees is broken. It makes some sense to determine the free energy of the system $F_{2}$ and the Landau Hamiltonian $H_{2}(\eta)$ in the Gaussian approximation on the basis of (6) and (26), by formulas

$$
\begin{gathered}
w_{2}(\eta) \equiv e^{\frac{F_{2}-H_{2}(\eta)}{T}}, \quad \int d \eta w_{2}(\eta)=1 ; \\
H_{2}(\eta)=\frac{T}{2} \sum_{a, b} D_{a b} \delta 1 F_{a} \delta \eta q_{b}, \quad F_{2}=T \ln \frac{\operatorname{det} D}{(2 \pi)^{s}}
\end{gathered}
$$

Our results show that Landau's idea [1] to use the effective Hamiltonian to calculate the total free energy of the system $F$ by the formula

$$
e^{-\frac{F}{T}}=\int d \eta e^{-\frac{H(\eta)}{T}}
$$

which follows from the normalization condition (3) and definition (6), is not realized. This is because our expression for the effective Hamiltonian (21) turns formula (28) into identity. At the same time, in the literature, formula (28) is widely used with a phenomenological effective Hamiltonian of the form 


$$
\begin{gathered}
H(\eta)=F_{0}(T, V)+\sum_{a_{1}} \alpha_{a_{1}}(T, V) \eta_{a_{1}}+\sum_{a_{1}, a_{2}} A_{a_{1} a_{2}}(T, V) \eta_{a_{1}} \eta_{a_{2}}+ \\
+\sum_{a_{1}, a_{2}, a_{3}} C_{a_{1} a_{2} a_{3}}(T, V) \eta_{a_{1}} \eta_{a_{2}} \eta_{a_{3}}+\sum_{a_{1}, a_{2}, a_{3}, a_{4}} B_{a_{1} a_{2} a_{3} a_{4}}(T, V) \eta_{a_{1}} \eta_{a_{2}} \eta_{a_{3}} \eta_{a_{4}} .
\end{gathered}
$$

(see, for example, $[2,12,13])$. It was proposed by Landau in his theory of phase transitions of the second kind for states in the vicinity of the phase transition point. The role of parameters $\eta_{a}$ in this case is played by the order parameter, which in this situation is small. Taking into account the system symmetry usually simplifies the coefficients in this expression.

It should be noted that our results (23) and (24) are complicated because they contain coefficients $A_{a}, A_{a b}$, which are expressed by formulas (17) and (21) through all equilibrium correlation functions of the system $\left\langle\hat{\eta}_{a_{1}} \ldots \hat{\eta}_{a_{n}}\right\rangle$ in the absence of the external field $h_{a}$. Exact calculation of the values $A_{a}, A_{a b}$ by the formulas (17) and (21) can only be done neglecting correlations $\left\langle\hat{\eta}_{a_{1}} \ldots \hat{\eta}_{a_{n}}\right\rangle$ with $n \geq 3$ and replacing $\mathbb{G}(i T u)$ by $\mathbb{G}_{2}(i T u)$

$$
\mathbb{G}_{2}(i T u)=-\frac{1}{2} \sum_{a, b} u_{a} u_{b} \mathbf{C}_{a b}, \quad \mathbf{C}_{a b} \equiv\left\langle\hat{\eta}_{a} \hat{\eta}_{b}\right\rangle
$$

In this approximation, the distribution function $w(\eta)$, according to (20), is given by an exact formula

$$
w_{2}(\eta)=\left[\frac{\operatorname{det} \mathbf{C}}{(2 \pi)^{s}}\right]^{1 / 2} e^{-\frac{1}{2} \sum_{a, b} \mathbf{C}_{a b}^{-1} \delta \eta_{a} \delta \eta_{b}}
$$

because

$$
\int d u e^{-(1 / 2) \sum_{a, b} \mathbf{C}_{a b} u_{a} u_{b}} e^{\sum_{a} i u_{a} \delta \eta_{a}}=\left[\frac{(2 \pi)^{S}}{\operatorname{det} \mathbf{C}}\right]^{1 / 2} e^{-(1 / 2) \sum_{a, b} \mathbf{C}_{a b}^{-1} \delta \eta_{a} \delta \eta_{b}} .
$$

The distribution (30) in comparison with (26) can be called a simplified Gaussian.

In our work [9] the problem of effective Hamiltonian realization in Landau's definition was considered in the macroscopic theory. Expression (23) for $H(\eta)$ was obtained in a more complex way than in the present paper, but no attention was paid to the validity of formula (24) for the distribution function $w(\eta)$. Also, no conclusion was made about the impossibility of calculating the total free energy of the system using formula (28) with the Landau Hamiltonian.

\section{Nonequilibrium Landau free energy}

The definition of the nonequilibrium thermodynamic potential of the system underlies the Landau theory of phase transitions of the second kind. Above we considered the description of the equilibrium state of the system in the presence of an external field $h_{a}$, which is introduced through the microscopic values $\hat{\eta}_{a}$ of some parameters $\eta_{a}$ in the form $\sum_{a} h_{a} \hat{\eta}_{a}$. The canonical Gibbs distribution function in such a situation $w(h)$ 
is given by formula (10). Consider in more detail the thermodynamics of such a system. Internal energy $E$, observed (average) value of the parameter $\eta_{a}$ and thermodynamic force $A_{a}$ corresponding to the external parameter $h_{a}$, elementary work $\delta R$ are given by standard formulas

$$
\begin{gathered}
E=\operatorname{Sp} w(h)\left(\hat{H}+\sum_{a} h_{a} \hat{\eta}_{a}\right), \quad \eta_{a}=\operatorname{Sp} w(h) \hat{\eta}_{a}, \\
A_{a}=-\operatorname{Sp} w(h) \frac{\partial}{\partial h_{a}}\left(\hat{H}+\sum_{a} h_{a} \hat{\eta}_{a}\right)=-\eta_{a}, \quad \delta R=\sum_{a} A_{a} d h_{a}=-\sum_{a} \eta_{a} d h_{a} .
\end{gathered}
$$

The basic relation of thermodynamics for the free energy $F(h)=E-T S$ follows from the equations of the first and second laws of thermodynamics

$$
\delta Q=d E+\delta R, \quad \delta Q=T d S
$$

and has the form

$$
d F(h)=-S d T+\sum_{a} \eta_{a} d h_{a} .
$$

Here we use the notation of free energy by $F(h)$ from expression (10), although free energy can be expressed in different thermodynamic variables too. At the same time, the field $h_{a}$ is its eigenvariable for the potential $F(h)$. According to Landau [1] (see also [4, 5]), the nonequilibrium thermodynamic potential $F_{L}(\eta)$ coincides with the equilibrium potential, which is given by the Legendre transformation

$$
F_{L}(\eta)=F(h)-\sum_{a} h_{a} \eta_{a} .
$$

Parameters $\eta_{a}$ are eigenvariables for this potential because

$$
d F_{L}(\eta)=-S d T-\sum_{a} h_{a} d \eta_{a}
$$

Finally, the nonequilibrium free energy of the system in the presence of an external field is given by the Landau formula

$$
F_{\text {neq }}(\eta, h)=F_{L}(\eta)+\sum_{a} \eta_{a} h_{a}
$$

where $\eta_{a}$ and $h_{a}$ are independent variables.

In the case of a small field $h_{a}$, the function $F_{L}(\eta)$ can be calculated explicitly [8]. First, formula (19) taking into account (17) gives the expansion of the free energy in the presence of a field $F(h)$ in a series in powers of the field $h_{a}$

$$
F(h)=F+\sum_{a} h_{a} \eta_{a 0}+\sum_{n=2}^{\infty} \frac{(-1)^{n-1}}{n ! T^{n-1}} \sum_{a_{1} \ldots a_{n}} h_{a_{1}} \ldots h_{a_{n}}\left\langle\hat{\eta}_{a_{1}} \ldots \hat{\eta}_{a_{n}}\right\rangle
$$

Then the basic thermodynamic relation (33) gives formula $\eta_{a}=\partial F(h) / \partial h_{a}$ and therefore from (38) expansion of $\eta_{a}$ in powers of the field

$$
\eta_{a}=\eta_{a 0}+\sum_{n=1}^{\infty} \frac{(-1)^{n}}{n ! T^{n}} \sum_{a_{1} \ldots a_{n}} h_{a_{1}} \ldots h_{a_{n}}\left\langle\eta_{a} \eta_{a_{1}} \ldots \eta_{a_{n}}\right\rangle .
$$


The potential $F_{L}$ as a function of the field $h_{a}$ according to (35), (38), and (39) has the form

$$
F_{L}=F+\sum_{n=2}^{\infty} \frac{(n-1)(-1)^{n}}{n ! T^{n-1}} \sum_{a_{1} \ldots a_{n}} h_{a_{1}} \ldots h_{a_{n}}\left\langle\hat{\eta}_{a_{1}} \ldots \hat{\eta}_{a_{n}}\right\rangle
$$

From formula (39) for small deviations $\delta \eta_{a}=\eta_{a}-\eta_{a 0}$ one can find an expression for the field $h_{a}$ through the deviations. Then formula (39) will give nonequilibrium free energy in the absence of an external field $F_{L}(\eta)$. In the Gaussian approximation we have

$$
\begin{gathered}
F_{L}(\eta)=F+\frac{T}{2} \sum_{a, b} \mathbf{C}_{a b}^{-1} \delta \eta_{a} \delta \eta_{b}+O\left(\delta \eta^{3}\right), \\
h_{a}(\eta)=-T \sum_{b} \mathbf{C}_{a b}^{-1} \delta \eta_{b}+O\left(\delta \eta^{2}\right), \quad \mathbf{C}_{a b}=\left\langle\hat{\eta}_{a} \hat{\eta}_{b}\right\rangle .
\end{gathered}
$$

It was noted above that in the literature [2] nonequilibrium free energy is identified with the effective Hamiltonian and therefore, according to Boltzmann's formula (5), the free energy (40) gives the probability density $w(\eta)$ that coincides with the simplified Gaussian distribution (30). Thus, Landau's definition of nonequilibrium free energy leads to the same result as Landau's theory of the effective Hamiltonian, if in the latter neglect all correlations except binary ones.

\section{Spatially inhomogeneous states}

When considering spatially inhomogeneous states, the role of parameters $\eta_{a}$ is played by local quantities $\eta_{i}(x)$ where $x_{n}$ is a point of three-dimensional space. Periodic boundary conditions are applied to all local quantities, assuming that the system is placed in a vessel $V$ in the form of a cube with an edge $V^{1 / 3}$. Arbitrary functions $f(x)$ are expanded into the Fourier series by formulas

$$
f(x)=\frac{1}{V} \sum_{k} f_{k} e^{i k x}, \quad f_{k} \equiv \int_{V} d^{3} x f(x) e^{-i k x},
$$

where the wave vector $k_{l}$ runs the value

$$
k_{l}=2 \pi n_{l} / V^{1 / 3}, \quad n_{l}=0, \pm 1, \pm 2, \ldots
$$

At the end of the calculations, the thermodynamic boundary transition should be performed, in which the sums are replaced by the integral according to the rule

$$
\sum_{k} \ldots \underset{V \rightarrow \infty}{\longrightarrow} \frac{V}{(2 \pi)^{3}} \int_{V} d^{3} k \ldots
$$

Long-wave fluctuations, which are responsible for the singular behavior of the observed quantities, are usually considered. In this case, the Fourier components $\delta \eta_{i k}$ of the parameter deviations $\delta \eta_{i}(x)$ from the equilibrium values are considered to be nonzero only for small $k_{l}$. In this case, the parameters $\delta \eta_{i}(x)$ describe weak 
inhomogeneous states. For arbitrary functions $f(x)$ and $g(x)$, according to definition (42), the formula is valid

$$
\int_{V} d^{3} x f(x) g(x)=\frac{1}{V} \sum_{k} f_{k} g_{-k} .
$$

Hence the following rules for the consideration of spatially inhomogeneous states, which show that this consideration is embedded in above results, are valid

$$
\begin{gathered}
a \rightarrow(i, x) \rightarrow(i, k), \quad \eta_{a} \rightarrow \eta_{i}(x) \rightarrow \eta_{i k}, \quad h_{a} \rightarrow h_{i}(x) \rightarrow h_{i k} ; \\
\sum_{a} \ldots \rightarrow \sum_{i} \int_{V} d^{3} x \ldots \rightarrow \frac{1}{V} \sum_{i, k} \ldots ; \quad \mathbf{C}_{a b} \rightarrow \mathbf{C}_{i i^{\prime}}\left(x, x^{\prime}\right) \rightarrow \mathbf{C}_{i i^{\prime}}\left(k, k^{\prime}\right) .
\end{gathered}
$$

Further these substitutions are used to analyze the Landau free energy $F_{L}(\eta)$ given by formula (41). It is convenient to return to formulas (39) and (40) that give

$$
\delta \eta_{a}=-\frac{1}{T} \sum_{a} \mathbf{C}_{a b} h_{b}+O\left(h^{2}\right), \quad F_{L}=F+\frac{1}{2 T} \sum_{a b} \mathbf{C}_{a b} h_{a} h_{b}+O\left(h^{3}\right)
$$

In spatially inhomogeneous states in terms of the Fourier components, these relations in accordance with (42) and (46) take the form

$$
\begin{gathered}
\delta \eta_{i k}=-\frac{1}{T} \frac{1}{V} \sum_{i^{\prime} k^{\prime}} \mathbf{C}_{i i^{\prime}}\left(k, k^{\prime}\right) h_{i^{\prime} k^{\prime}}^{*}+O\left(h^{2}\right), \\
F_{L}=F+\frac{1}{2 T} \frac{1}{V^{2}} \sum_{i k i^{\prime} k^{\prime}} \mathbf{C}_{i i^{\prime}}\left(k, k^{\prime}\right) h_{i k}^{*} h_{i^{\prime} k^{\prime}}^{*}+O\left(h^{3}\right) .
\end{gathered}
$$

Further the equilibrium state of the system in the absence of the external field is considered to be spatially homogeneous. In this case, the correlation functions are translationally invariant and therefore we have consistently

$$
\begin{aligned}
& \mathbf{C}_{i i^{\prime}}\left(k, k^{\prime}\right)=\left\langle\hat{\eta}_{i k} \hat{\eta}_{i^{\prime} k^{\prime}}\right\rangle=\int_{V} d^{3} x \int_{V} d^{3} x^{\prime}\left\langle\hat{\eta}_{i}(x) \hat{\eta}_{i^{\prime}}\left(x^{\prime}\right)\right\rangle e^{-i k x-i k^{\prime} x^{\prime}}= \\
& =\int_{V} d^{3} x \int_{V-x} d^{3} x^{\prime \prime}\left\langle\hat{\eta}_{i}(x) \hat{\eta}_{i^{\prime}}\left(x^{\prime \prime}+x\right)\right\rangle e^{-i\left(k+k^{\prime}\right) x-i k^{\prime} x^{\prime \prime}} \underset{V \rightarrow \infty}{=} \\
& =\int_{V} d^{3} x e^{-i\left(k+k^{\prime}\right) x} \int d^{3} x^{\prime \prime}\left\langle\hat{\eta}_{i}(0) \hat{\eta}_{i^{\prime}}\left(x^{\prime \prime}\right)\right\rangle e^{-i k^{\prime} x^{\prime \prime}}=V \delta_{k^{\prime},-k} \mathbf{C}_{i i^{\prime}}(k), \\
& \mathbf{C}_{i i^{\prime}}(k) \equiv \int d^{3} x\left\langle\hat{\eta}_{i}(0) \hat{\eta}_{i^{\prime}}(x)\right\rangle e^{i k x}, \quad \mathbf{C}_{i i^{\prime}}^{*}(k)=\mathbf{C}_{i^{\prime} i}(k)
\end{aligned}
$$

( $\left\langle\hat{\eta}_{i}(0) \hat{\eta}_{i^{\prime}}(x)\right\rangle=0$ when $\left|x-x^{\prime}\right| \gg>R_{\text {cor }} ; R_{\text {cor }}$ is a radius of equilibrium correlations).

Taking into account (49), from formulas (48) the expression for the nonequilibrium Landau free energy in the spatially inhomogeneous state of the system is obtained.

$$
F_{L}=F+\frac{T}{2} \sum_{i i^{\prime}} \frac{1}{V} \sum_{k} \mathbf{C}_{i i^{\prime}}^{-1}(k) \delta \eta_{i k}^{*} \delta \eta_{i^{\prime} k}+O\left(\delta \eta^{3}\right)
$$


Since fluctuations, for which quantities $\delta \eta_{i k}$ are non-zero only for small $k_{l}$, are considered, function $\mathbf{C}_{i i^{\prime}}^{-1}(k)$ can be expanded in a series in powers of $k_{l}$. The structure of such a series depends on the symmetry of the equilibrium state of the system and in the simplest case has the form

$$
\mathbf{C}_{i i^{\prime}}^{-1}(k)=2\left(a \delta_{i i^{\prime}}-g \delta_{i i^{\prime}} \delta_{n l} k_{n} k_{l}\right) / T
$$

( $a, g$ are some real functions of the temperature), which gives the nonequilibrium free energy of the form

$$
F_{L}=F+\int_{V} d^{3} x\left[a \sum_{i} \delta \eta_{i}(x)^{2}+g \sum_{i} \frac{\partial \delta \eta_{i}(x)}{\partial x_{l}} \frac{\partial \delta \eta_{i}(x)}{\partial x_{l}}\right] .
$$

Contributions of this kind to nonequilibrium free energy are used in the theory of phase transitions of the second kind to study long-wave fluctuations of the order parameter (see, for example, $[1,2,10,11])$.

\section{Conclusions}

For an arbitrary system described by classical mechanics, a microscopic theory has been developed to calculate the probability density $w(\eta)$ of the values of some set of parameters $\eta_{a}$, which give the reduced description of fluctuations in the system in the vicinity of the equilibrium state. To do this, the approach of our previous work [9] is simplified and $w(\eta)$ is expressed through all equilibrium correlation functions of the parameters $\eta_{a}$. The expression for $w(\eta)$ in microscopic theory, but only through binary correlation function, was previously obtained by Leontovich [6, 7]. The definition of the Landau effective Hamiltonian of the system $H(\eta)$ is discussed. It is found that this definition gives an expression for $H(\eta)-F$ (where $F$ is the exact free energy of the system) and does not allow calculating $F$ through the statistical integral corresponding to the Landau Hamiltonian $H(\eta)$.

For an arbitrary system, which is described by classical mechanics, a microscopic realization of the Landau definition of the nonequilibrium free energy of the system is developed for a state having a reduced description with some set of parameters $\eta_{a}$. This free energy is an equilibrium thermodynamic potential, the eigenvariables of which are parameters $\eta_{a}$. We introduced this potential through the Legendre transformation of the free energy of the system in the presence of an external field. There is no clear reference to such a procedure in Landau's works (see [1, 3, 4]), but [5] states that it is his idea. Somewhat later, a similar definition of nonequilibrium free energy was proposed by Leontovich [6,7], but also without its microscopic realization. In the literature, nonequilibrium free energy is identified with the effective Hamiltonian [2]. We have established that the Landau nonequilibrium free energy as an effective Hamiltonian, when used in the Boltzmann formula, gives an expression for the fluctuation distribution function $w(\eta)$ that takes into account only binary correlations of the parameters $\eta_{a}$.

On the example of the Landau nonequilibrium free energy it is shown that the description of fluctuations in spatially inhomogeneous states can be included into the developed here theory. In terms of the microscopic theory, the origin of the contributions 
to free energy, which depend on the gradients of the parameters describing the fluctuations in the system, is clarified.

\section{References}

1. Landau, L.D. Statistical Physics. Part 1 / L.D. Landau, E.M. Lifshitz. - Oxford: Pergamon Press, 1980. - 544 p.

2. Patashinskii, A.Z. Fluktuatsionnaya teoriya fazovykh perekhodov / A.Z.

Patashinskii, $\quad$ V.L. Pokrovskii. - M.: Nauka, 1982. - 382 p.

3. Landau, L.D. K teorii fazovykh perekhodov. I / L.D. Landau, E.M. Lifshitz // ZhETF. - 1937. - Vol. 7, Issue 19. - P. 627-635.

4. Landau, L.D. Elektrodinamika sploshnykh sred / L.D. Landau, E.M. Lifshitz. M.: Nauka, 1982. -620 p.

5. Rumer, Yu.B. Termodinamika, statisticheskaya fizika i kinetika / Yu.B. Rumer, M.Sh. Ryvkin. - M.: Nauka, 1977. - 552 p.

6.. Leontovich, M.A. O svobodnoy energii neravnovesnogo sostoyaniya / M.A. Leontovich // ZhETF. - 1938. - Vol. 8, Issue 7. - P. 844-854.

7. Leontovich, M.A. Izbrannye trudy. Teoreticheskaya fizika / M.A. Leontovich. M.: Nauka, 1985. -432 p.

8. Haponenko, K.M. Non-equilibrium Gibbs thermodynamic potential of a magnetic system / K.M. Haponenko, A. Sokolovsky // Visnyk Dnipropetrovs'koho unìversitetu. Seriya Fizyka, radioelektronika. - 2016. - Vol. 24, Issue 23. - P. 45-52.

9. Haponenko, K.M. Landau effective Hamiltonian and its application to the magnetic system / K.M. Haponenko, A.I. Sokolovsky // Journal of Physics and Electronics. - 2018. - Vol. 26, No. 1. - P. 19-28.

10. Ma, Sh. Modern theory of critical phenomena / Sh. Ma. - New York, Westview Press, 2000. $-588 \mathrm{p}$.

11. Lebedev, V.V. Fluktuatsionnyye efekty v makrofizike / V.V. Lebedev. - M.: MTSMNO, 2004. - $256 \mathrm{p}$.

12. Yukhnovskii, I.R. Fazovi perekhody v okoli krytychnoi tochky haz-ridyna / I.R. Yukhnovsky // Ukr. Journ. of Physics. Reviews. - 2015. - Vol. 10, No. 1. - P. 33-97.

13. Akhiezer, A.I. Methods of Statistical Physics / A.I. Akhiezer, S.V. Peletminskii. - Oxford: Pergamon Press, 1981. - 450 p.

14. Stratonovich, R.L. Nelinejnaya neravnovesnaya termodinamika / R.L. Stratonovich. - M.: Nauka, 1985. - 480 p. 